\title{
A Water Monitoring and Early Warning System of COD Using UV-Vis Spectroscopy
}

\author{
Tang Yuan ${ }^{1}$, Wei Biao ${ }^{1}$, Wu De-chao ${ }^{1,2}$, Liu Juan ${ }^{1}$, Xiong Shuang-fei ${ }^{1}$, Zhang Zhen ${ }^{1}$, Tang Ge ${ }^{1}$, \\ Feng Peng ${ }^{1}$
}

${ }^{1}$ College of Optoelectronic Engineering, Chongqing University, Chongqing, China

${ }^{2}$ College of Automation,Chongqing Industry Polytechnic College, Chongqing, China

\section{Email address:}

tangyuan@cqu.edu.cn (Tang Yuan),weibiao@cqu.edu.cn (Wei Biao), wudecao@qq.com (Wu De-chao), 466860935@qq.com (Liu Juan), 1215313597@qq.com (Xiong Shuang-fei),475571772@qq.com (Zhang Zhen),158148957@qq.com (Tang Ge), 30289927@qq.com (Feng Peng)

\section{To cite this article:}

Tang Yuan, Wei Biao, Wu De-chao, Liu Juan, Xiong Shuang-fei, Zhang Zhen, Tang Ge, Feng Peng. A Water Monitoring and Early Warning System of COD Using UV-Vis Spectroscopy. International Journal of Environmental Monitoring and Analysis.

Vol. 3, No. 5, 2015, pp. 298-303. doi: 10.11648/j.ijema.20150305.19

\begin{abstract}
A distributed remote water monitoring and early warning system was proposed, which used the UV-Vis spectroscopy, GPS, GIS, 4G network, COD solution and database technology, to solve the problems including long detection cycle, limited scale and the lack of early warning mechanism of the water monitoring. 50 groups of original water sample from Ciqikou to Chaotianmen of Jialing River in Chongqing, China (every $5 \mathrm{~km}$ decorate a monitoring node, every 60 minutes collect data) was selected to study distributed remote water monitoring and early warning system of COD in UV-Vis spectroscopy based on $4 \mathrm{G}$ networks. The results indicated that through the system, not only the real-time display and storage, historical data query and warning function of water quality monitoring information could be achieved, but also the status of the water quality environment on the basis of COD can be reflected, which has a strong practicability and can provide environmental monitoring departments with real-time water quality information.
\end{abstract}

Keywords: Water Quality Monitoring of COD, UV-Vis Spectrometry, 4G Networks, Early Warning

\section{一种紫外-可见光谱水体COD监测预警系统}

\author{
唐媛 ${ }^{1}$ 魏彪 ${ }^{1}$, 吴德操 ${ }^{1,2}$, 刘娟 ${ }^{1}$, 熊双飞 ${ }^{1}$, 张峥 ${ }^{1}$, 汤戈 ${ }^{1}$, 冯鹏 ${ }^{1}$ \\ ${ }^{1}$ 光电工程学院, 重庆大学, 重庆, 中国 \\ ${ }^{2}$ 自动化学院, 重庆工业职业技术学院, 重庆, 中国 \\ 邮箱 \\ tangyuan@cqu.edu.cn (唐媛),weibiao@cqu.edu.cn(魏彪), wudecao@qq. com(吴德操), 466860935@qq. com(刘娟), \\ 1215313597@qq. com(熊双飞), 475571772@qq. com(张峥), 158148957@qq. com(汤戈), 30289927@qq. com (冯鹏)
}

中文摘要: 针对目前水质环境检测周期长、水域范围受限及缺乏水质变化预警机制等问题，以紫外一可见光谱法水体COD 检测为手段，利用GPS、GIS、4G网络、COD解算以及数据库等技术，提出了一种分布式远程水质环境监测预警系统。选 取中国重庆主城区嘉陵江流域的磁器口到朝天门水域段, 每隔 $5 \mathrm{~km}$ 布置一个监测节点, 每 60 分钟进行一次 50 组原始水体 水样数据采集, 开展了基于 $4 \mathrm{G}$ 网络的分布式远程紫外-可见光谱水体COD监测预警系统研究。研究结果表明, 系统不仅 实现了水体水质监测信息实时显示与存储、历史数据查询以及预警等功能, 而且依据水体COD可以实时、在线反映水质 环境的状况, 此可为环境监测部门提供水体实时监测信息, 具有很强的实用性。

关键词: 水体COD监测, 紫外-可见光谱法, 4G网络, 预警 


\section{1. 引言}

众所周知, 随着中国社会和经济的快速发展, 触发了 工业废水和城市生活污水的大量排放, 致使原本匮乏的地 表水资源受到极大的污染, 敲响了饮用水水质安全的警钟。 为此, 人们需要对水体水质的安全性进行在线、实时、全 天候、整流域的监测预警, 以此防范地表水资源不被受到 污染, 确保人们日常生活饮用地表水水质的安全性。

目前, 常用的水体监测技术主要有化学或电化学分析、 色谱分析以及光谱分析等方法 [1]。然而, 此类水体监测 技术以人工采集水样在实验室进行手工分析为主, 难以做 到对水体在线、实时性的监测要求。即便较为成熟的水体 监测化学分析法也至少需要20分钟才能得出检测结果, 无 法为水体污染事件的发生提供及时的预警。

基于紫外 - 可见光光谱 (ultraviolet-visible spectrometry, UV-Vis）分析技术可以直接或间接测定水 体中大多数金属离子、非金属离子、有机物污染物、硝酸 盐以及化学需氧量 (Chemical Oxygen Demand, COD) 等 多种参数。其中, 化学需氧量 (COD) 反映了水体中还原性 物质的污染程度, 它是评价水质环境的一个重要指标。 UV-Vis 光谱法监测水体COD就是利用测得水体的紫外-可 见吸收光谱来建立水质相关的参数模型, 即建立水体 $\mathrm{COD}$ 与其紫外-可见光吸收光谱之间的对应关系, 该法具有无 需化学试剂、测量速度快等优点 $[2,3]$ 。同时, UV-Vis 光谱法水体监测, 可以将光谱探头浸入水中, 能够对水体 进行在线、实时的监测, 从而实现对水体环境实时监控、 突发性污染事故预警以及流域趋势分析等功能。特别是, 随着传输速率快、频带宽的 $4 \mathrm{G}$ 通信技术的迅猛发展, 全天 候、整流域水体安全性监测成为可能, 此可将水体是否发 生污染险情上传至监控中心, 从而及时发出预警信息, 预 先做好相应的预防措施, 以便将损失降到最低。

有鉴于此, 本文提出了一种基于4G网络的分布式远程 紫外-可见光谱水体 COD监测预警系统。该系统借助现有的 移动通信网络, 将水体光谱法监测节点信息通过 $4 \mathrm{G}$ 网络传 送至远程监控中心服务器, 实现对水体COD的在线测量、 实时监测、突发性预警、流域分析以及水环境监控, 减少 因水体污染造成的损失，保障人们饮水安全。

\section{UV-Vis光谱法水体COD解算原理}

UV-Vis 光谱法监测水体 COD, 实质上是构建基于 UV-Vis光谱法的水体COD预测模型 $[4,5]$ 。它是以水样的 光谱数据与水体 $\mathrm{COD}$ 间的数学模型为基础, 将待测水样的 光谱数据导入所建模型, 预测出其相应的水体COD值。因 此, 建模方法的合理选取决定了水体 COD预测模型的精准 度。

目前, 代表性的建模方法有偏最小二乘、神经网络、 支持向量机 (SVM) 以及最小二乘支持向量机 (LSSVM) 等算 法, 其中LSSVM较一般的SVM算法预测精度高、计算简单。 其基本原理 [6] 为：对于给定的训练数据集 $\left(x_{i}, y_{i}\right), i=1,2, \ldots n$ 。 $x_{i}=R^{n}$ 为 $\mathrm{n}$ 维输入向量, $y_{i}=R$ 为 一维输出向量, $\mathrm{n}$ 为样本数。在高维特征空间中可构造最 优决策函数式 (1) 所示。

$$
y=\omega \cdot \varnothing(x)+b
$$

式 (1) 中, $\omega \in R^{n}$ 为权向量, $\varnothing(x)$ 是将输入向 量映射到高维特征空间的函数。

利用结构风险最小化原理, 最小化目标函数及其约束 条件可表示为公式 (2) 所示。

$$
\left\{\begin{array}{l}
\min J(\omega, \xi)=\frac{1}{2} \omega^{T} \omega+C \sum_{i=1}^{n} \xi_{i}^{2} \\
y_{i}=\varnothing\left(x_{i}\right) \cdot \omega+b+\xi_{i}, i=1,2, \ldots n
\end{array}\right.
$$

式 (2) 中, C为正规化参数, 控制误差样本的惩罚程 度; $\xi_{i}$ 为误差变量; $\mathrm{b}$ 为偏置参数。

利用拉格朗日法求解式 (2), 可得公式 (3) 所示。

$$
L(\omega, b, \xi, \alpha)=\frac{1}{2} \omega^{T} \omega+C \sum_{i=1}^{n} \xi_{i}^{2}-\sum_{i=1}^{n} \alpha_{i}\left[\varnothing\left(x_{i}\right) \cdot \omega+b+\xi_{i}-y_{i}\right]
$$

式 (3) 中, $\alpha_{i}(i=1,2, \ldots n)$ 为拉格朗日乘子。定义 核函数 $K\left(x_{i}, x_{j}\right)=\varnothing\left(x_{i}\right) \cdot \varnothing\left(x_{j}\right)$, 可将式 (3) 转化为线性 方程组式 (4) 所示。

$$
\left[\begin{array}{cccc}
0 & 1 & \cdots & 1 \\
1 & K\left(x_{1}, x_{1}\right)+\frac{1}{C} & \cdots & K\left(x_{1}, x_{n}\right) \\
\vdots & \vdots & \ddots & \vdots \\
1 & K\left(x_{n}, x_{1}\right) & \cdots & K\left(x_{n}, x_{n}\right)+\frac{1}{C}
\end{array}\right] \bullet \cdot\left[\begin{array}{c}
b \\
\alpha_{1} \\
\vdots \\
\alpha_{n}
\end{array}\right]=\left[\begin{array}{c}
0 \\
y_{1} \\
\vdots \\
y_{n}
\end{array}\right]
$$

最后, 利用最小二乘法求解式 (4), 可得出回归系数 $a$ 和偏差 $b$ 。由此, 得出非线性预测模型式 (5) 所示。

$$
y_{i}=\sum_{i=1}^{n} \alpha_{i} K\left(x, x_{i}\right)+b
$$

式 (5) 中, $x_{i}$ 为待测样本的光谱数据, $y_{i}$ 为待测样 本的预测COD值。

\section{3. 基于 $4 \mathrm{G}$ 网络的光谱法水体 $\mathrm{COD}$ 监测预警系统}

\section{1. 系统总体架构设计}

为满足水体分布式监测预警系统智能化与自动化的 实际应用要求, 该系统采用 $\mathrm{C} / \mathrm{S}$ 构架, 由水体监测节点、 4G无线通信网络以及远程监控中心三部分组成。系统原理 结构, 如图1所示。 


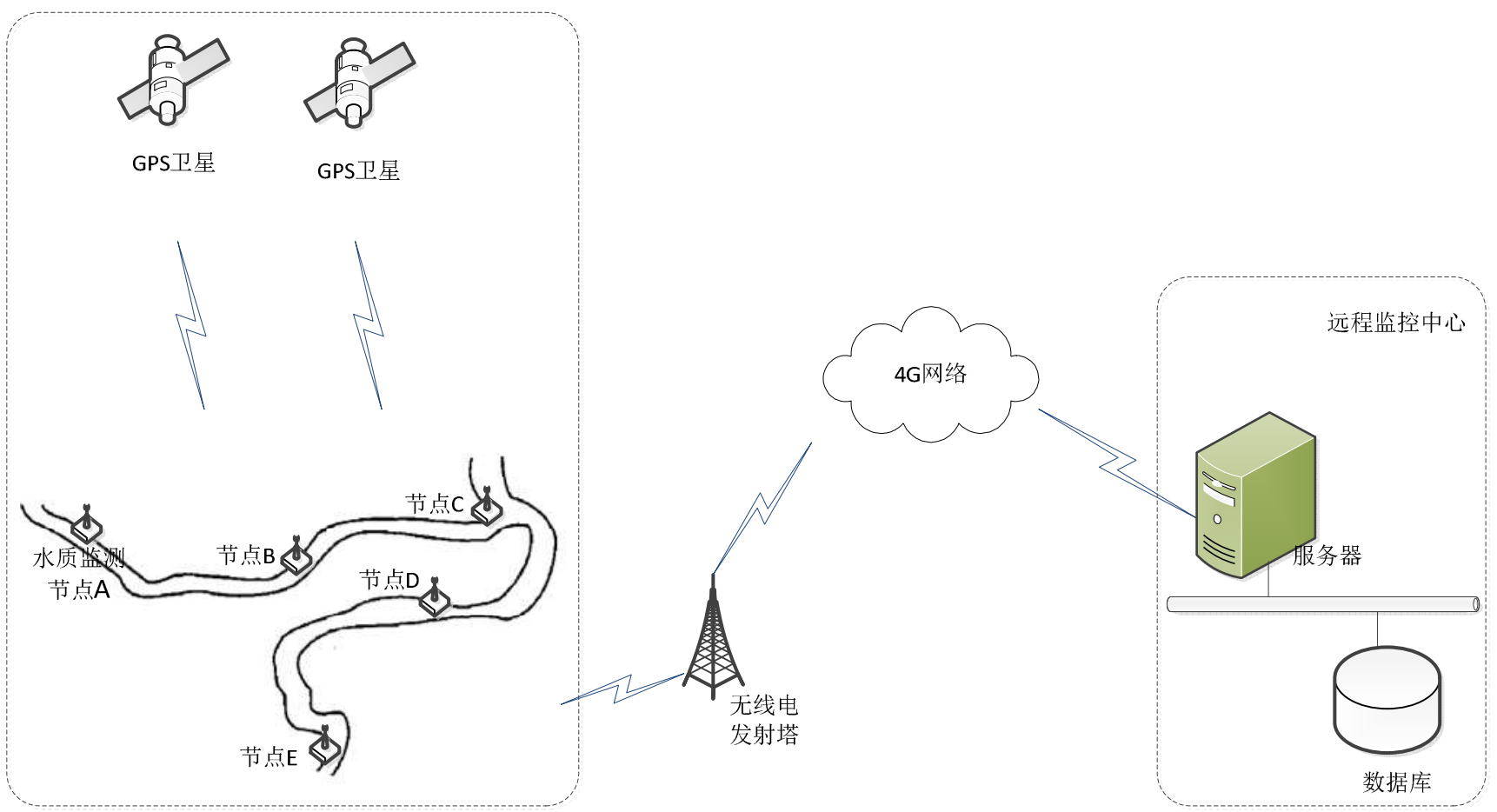

图1 分布式远程水体监测预警系统原理图。

基于 $4 G$ 网络的分布式远程紫外-可见光谱水体监测预 警系统，它是一个一对多的远程无线双向数据通信和控制 系统。任意分布在流域中的光谱浸入式探头水体监测节点, 通过紫外-可见光谱仪、传感器等设备进行水质数据的实 时采集, GPS模块获取节点地理位置信息; 利用 $4 \mathrm{G}$ 无线网 络将数据信息发送至远程具有静态IP地址或支持DDNS (动 态域名解析) 的监控中心服务器, 以此进行水体水质监测 数据的存储、分析、显示、查询以及管理等操作 [7]。远 程监控中心通过GIS系统可清楚、直观地查看整个流域中 水体水质变化情况以及节点设备的运行情况。同时, 可对 各监测节点发送控制指令, 各节点根据指令要求进行相应 操作。

\section{2. 水体监测节点设计}

\subsection{1. 硬件设计}

水体监测节点由紫外-可见光谱仪、GPS定位模块、4G 无线模块以及其他辅助传感器 ( PH、温度、电导率) 等构 成, 完成水体水质信息的原位采集、变换、传输等操作 [8-10], 其结构框图如图2所示。系统主控芯片采用基于 ARM Cortex-A7 CPU和 Mali400MP2 GPU架构的全志A20双 核处理器, 该芯片支持1GB的高速DDR3内存和16GB存储容 量。该系统稳定流畅、超低功耗、接口丰富、集成度高, 非常适合在控制领域应用。

4G无线网络是基于IP协议的高速蜂窝移动网, 其传输 速率达到100Mbit/s。4G模块借用移动通信网络的接入网 和传输网, 提供端到端的无线IP连接, 实现水体监测节点 与监控中心的通讯连接。它具有建设周期短、传输速率快
以及传输视频质量高等特点 [11], 能够实现监控中心 24 小时全方位对流域监测节点及其周围环境的监控, 大幅度 提高了系统在移动性、便捷性等方面的综合功能。

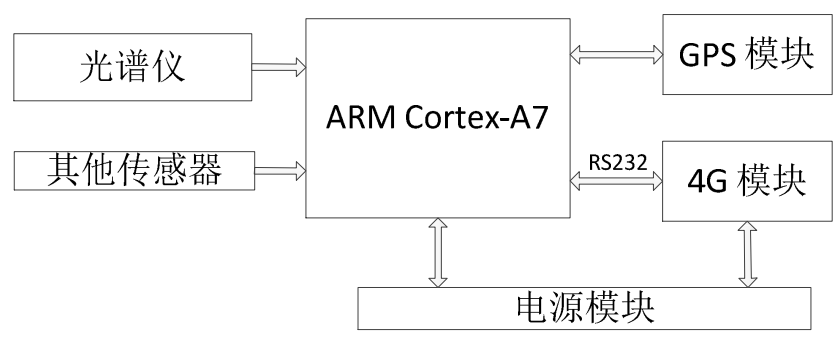

图2 水体监测节点硬件结构图。

此外, 由于 $4 \mathrm{G}$ 无线网络的使用资费以传输资料单位计 算, 故在数据传输之前将用压缩感知算法对水质数据压缩, 减少因数据流量产生的费用，同时提高传输效率。

\subsection{2. 软件设计}

水体监测节点主要任务是完成水体水质参数的检测、 数据的传输、短期数据的存储以及接受监控中心的指令。 鉴于其长期处于野外环境, 因此选用低功耗、高性能的 Linux操作系统搭建软件平台。

水体监测节点上电以后, 首先, 初始化用于区分各监 测节点的ID号、定时采集周期以及休眠时间等信息; 然后, 根据预先设定在其内部的IP地址, 主动地访问网络代理服 务器, 通过代理服务器与监控中心建立TCP/IP链路。当监 控中心要向某个监测节点提出数据请求时, 它会根据IP 
地址和ID号来找到对应的节点下达命令。其软件实现流程, 如图3所示。

由于水体监测节点使用太阳能电池供电, 因而节点采 用低功耗的休眠/唤醒机制 [12], 由处理器分配数据定时 采集时间。监测节点在不进行数据采集时, 进入睡眠模式, 每次休眠醒来之后, 查询是否有操作指令, 如果有指令, 则进行相应操作; 如果定时采集时间到达, 执行数据采集 和传输, 数据发送完毕后, 又立即进入睡眠模式。

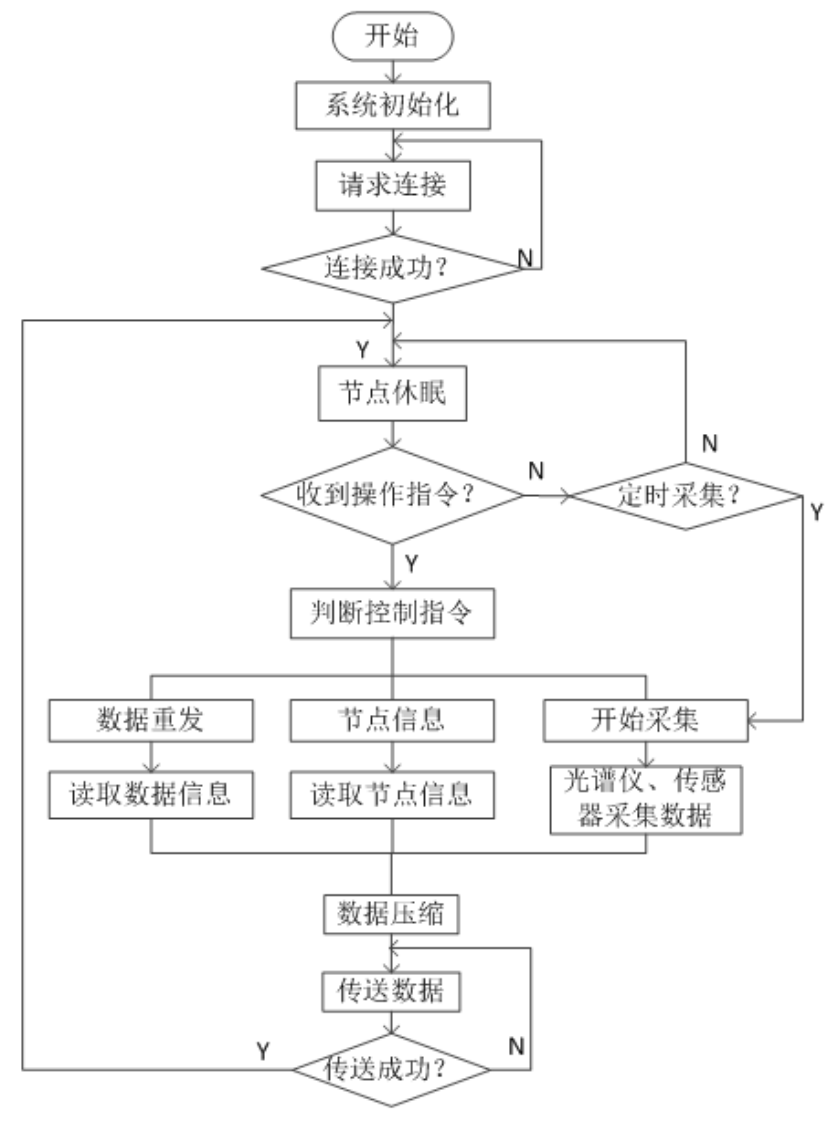

图3 水体监测节点软件流程图。

\section{3. 监控中心设计}

\subsection{1. 软件设计}

远程监测中心服务器综合应用GIS、数据库以及曲线绘 制等技术 [13]，可长期、实时接收监测节点的水体水质光 谱数据和常规参数, 通过数据库进行管理和存储, 实现水 体监测数据的实时显示与分析、历史数据查询以及节点信 息的显示等功能, 并可向节点发送参数设置、工作启停等 指令。研究中, 以Microsoft Visual C++为开发平台, Microsoft SQL Server 2005作为后台数据库实现节点数据 的存储和读取, 结合ESRI公司的Arc GIS Engine嵌入式组 件库, 实现节点地理位置信息的实时显示 $[14,15]$ 。利用 Chart控件绘制水质数据的动态曲线, 直观了解水质数据的 变化趋势, 对突发性的水体水质安全性与否进行快速预警。

\subsection{2. 心跳包机制}

心跳包机制是监控系统在无法确保网络可靠性的前 提下, 客户端每隔固定时间发送一个特定心跳数据包信息 给服务器, 通知服务器自己仍然在线, 服务器收到后回复 一个相应信息。如果服务器在指定时间内没有收到客户端 信息，则认为客户端断开; 同时，如果客户端在指定时间 内没有收到服务器返回的信息, 则认为网络异常中断, 并 采取相应的措施 [16]。其基本流程，如图4所示。

水体监测节点客户端与远程监控中心服务器建立连 接后, 监测节点进入低功耗状态, 并周期性地向监控中心 服务器发送心跳包数据。一旦服务器接收到心跳包数据, 就返回一个校验数据包给检测节点, 表示连接正常; 否则 认为连接出现问题, 节点将重新连接服务器。如果在指定 的连接次数内连接成功, 则认为监测节点和监控中心连接 正常, 可继续进行数据的通信; 反之, 则认为连接中断。

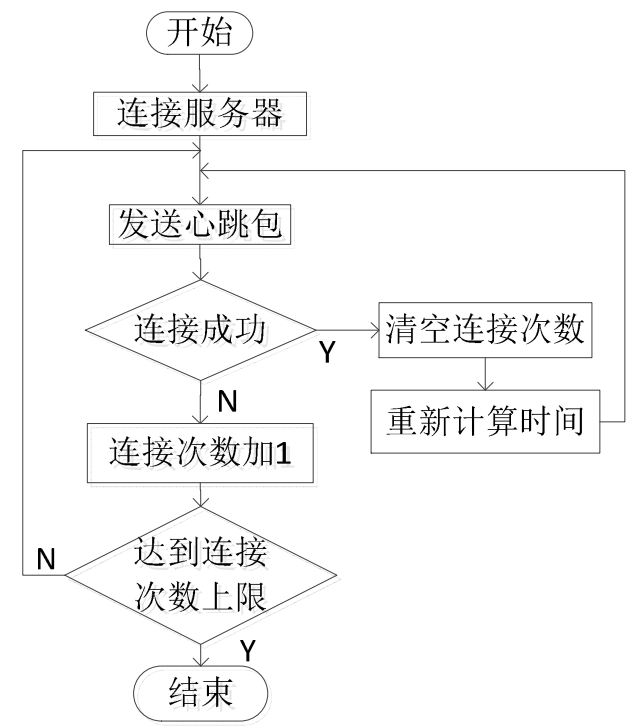

图4 心跳包流程图。

\section{4. 实验结果与分析}

为了测试该监控预警系统的综合性能, 对中国重庆主 城区嘉陵江流域的磁器口到朝天门水域内水体水质进行 数据的采集、传输、显示以及预警等试验。在该流域段, 每隔 $5 \mathrm{~km}$ 布置一个监测节点, 每 60 分钟进行一次数据采集, 休眠时间设置为5分钟。

实验中, 选取监测流域内 50 组原始水体水样, 用蒸馏 水按 9 种不同比例进行稀释, 将稀释后的500组水体水样作 为训练样本。将每组水样的吸收光谱与化学法实测出的 COD值进行LSSVM-COD解算模型训练, 经过多次训练得到较 高精度的预测模型, 然后将预测水体水样吸收光谱数据输 入模型, 可得到精度较高的COD值。监控人员可通过实时 光谱功能查询得到监测节点在某时刻的吸收光谱曲线以 及通过模型解算出的COD值, 如图5为嘉陵江生活污水排污 口（监测节点1）在2015年4月9日19:45的实时光谱及对应 的COD值。 
系统拥有查询历史数据、曲线显示等功能, 监控人员 可通过选择监测节点和起始时间、终止时间来获得该节点 的COD、PH值、水温等水质信息。图6（a）为监测节点 1 在 2015 年 4 月 9 日当天的各项水质数据。通过查询历史曲线 功能, 可方便、直观地反映在该时间段内, 监测节点水质 的变化趋势。设置水质参数的预警值, 可形象地观察出所
查询水质参数的超标时段, 监控人员可根据查询结果对该 时段的水质状况进行进一步的分析和管理。图6（b）为监 测节点 1 在 2015 年 4 月 9 日当天水体COD的变化曲线, 当COD 值超过预设的 $40 \mathrm{mg} / \mathrm{L}$ 时, 曲线变为红色, 便于监控人员直 观分析。

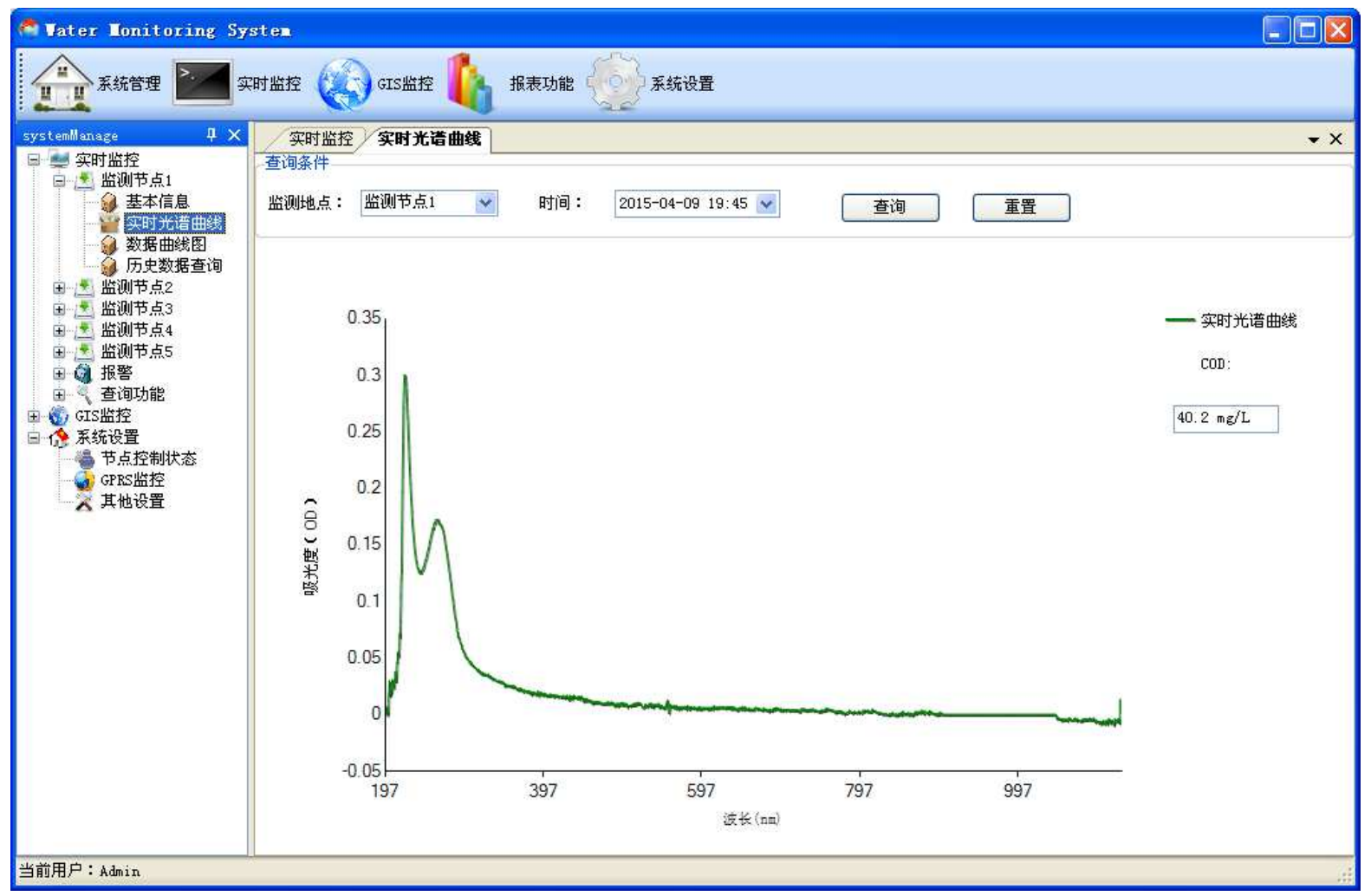

图5 远程水质监控中心实时光谱曲线及其COD值。

\begin{tabular}{|c|c|c|c|c|c|c|c|c|}
\hline \multirow[t]{2}{*}{ 监测地点 } & \multicolumn{2}{|c|}{ : 监测节点 1 } & \multirow[t]{2}{*}{ 起始时间： } & \multicolumn{2}{|c|}{ 2015-04-0900:00 $\mathrm{V}$} & 终止时间： & \multicolumn{2}{|c|}{ 2015-04-09 23:00 $\mathrm{V}$} \\
\hline & & & & & & 亘询 & & 重置 \\
\hline & 日期 & 时间 & 检测节点 & $\cos (m / L)$ & PH值 & $\begin{array}{l}\text { 水温 } \\
(\mathrm{oc})\end{array}$ & $\begin{array}{l}\text { 浊席 } \\
\text { (irvu) }\end{array}$ & 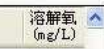 \\
\hline & $2015-4-9$ & $05: 00$ & 监测节点1 & 34.2 & 8. 28 & 15.8 & 0.29 & 8.46 \\
\hline & $2015-4-9$ & $06: 00$ & 监测节点1 & 32.5 & 8. 29 & 15.6 & 0.30 & 8. 44 \\
\hline & $2015-4-9$ & $07: 00$ & 监测节点1 & 28.1 & 8.28 & 16.0 & 0.28 & 8.43 \\
\hline & $2015-4-9$ & $08: 00$ & 监测节点1 & 22.6 & 8.26 & 16.1 & 0.26 & 8.45 \\
\hline & $2015-4-9$ & $09: 00$ & 监测节点1 & 23.3 & 8. 25 & 16.3 & 0.25 & 8.42 \\
\hline & $2015-4-9$ & $10: 00$ & 监测节点1 & 23.9 & 8.27 & 16.5 & 0.27 & 8.40 \\
\hline & $2015-4-9$ & $11: 00$ & 监测节点1 & 24.7 & 8. 30 & 16.9 & 0.30 & 8. 39 \\
\hline & $2015-4-9$ & $12: 00$ & 监测节点1 & 25.9 & 8. 35 & 17.6 & 0.31 & 8.44 \\
\hline & $2015-4-9$ & $13: 00$ & 监测节点1 & 26.3 & 8.34 & 17.8 & 0.29 & 8.47 \\
\hline$\leq$ & & & & iti & & & & $>$ \\
\hline
\end{tabular}

(a) 水质参数历史数据查询

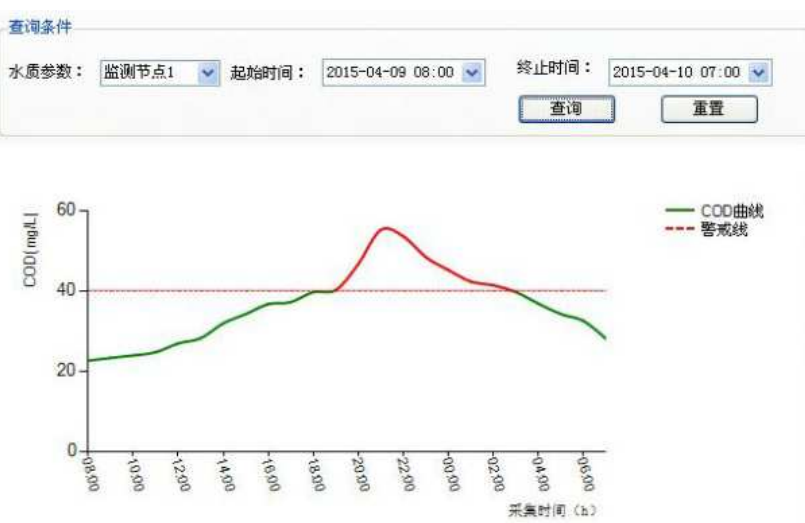

（b）水体COD历史数据曲线

图6 远程水质监控中心监测节点查询历史数据。

远程监控系统, 可减少水域监控的人力、物力等资源 的浪费。系统全天候的监测、实时显示功能, 可获得监控 流域的实时水质信息, 便于对流域水质进行分析管理; 历 史数据查询、超标预警功能, 使得系统对污染水质能够做 出快速响应, 减小水质污染带来的严重影响。

\section{5. 结束语}

本文开展了基于 $4 \mathrm{G}$ 网络的分布式远程紫外-可见吸收 光谱法水体COD监测预警系统研究。利用Visual C++编写 的数据解算及节点监控系统, 不仅实现了水体水质监测信 息实时显示与存储、历史数据查询以及超标预警等功能, 
而且可以实时、在线反映水质环境的状况（COD反映了水 体中还原性物质的污染程度），此可为环境监测部门提供 水质实时监测信息。通过对中国重庆嘉陵江流域实测结果 表明: 该系统运行稳定, 水体监测节点能够实现在无人监 控的情况下水体COD参数的自动采集、可靠传输; 监控中 心可以对流域水体水质数据进行实时显示、可视化分析、 历史数据查询以及超标预警等功能。同时, 该系统部署方 便、维护便捷, 可望获得广阔的应用前景。

\section{致谢}

本文研究工作得到了中国四川省科技支撑计划资助 项目《多功能水质实时自动监测技术开发与产品研制》 (2012SZ0111) 和中国重庆市研究生科研创新项目《基于无 线传感网络的光谱水质监测系统》 (CYS14039) 的资助。

\section{参考文献}

[1] Storey M V, van der Gaag B, Burns B P. Advances in on-line drinking water quality monitoring and early warning systems $[J]$. Water Research. 2011, $45(2)$ : 741-747.

[2] 魏康林, 温志渝, 武新, 等. 基于紫外-可见光谱分析的水 质监测技术研究进展 $[\mathrm{J}]$. 光谱学与光谱分析. 2011 (04) : 1074-1077.

[3] 侯迪波, 张坚, 陈冷, 等. 基于紫外-可见光光谱的水质分 析方法研究进展与应用 $[\mathrm{J}]$. 光谱学与光谱分析. $2013(07)$ : 1839-1844.

４4］汤斌, 赵敬晓, 魏虎, 等. 一种紫外-可见光谱检测水质COD 预测模型优化方法 [J]. 中国环境科学. 2015 (02) : 478-483.

[5] 孙荣霞, 王丽华, 张俊林. 支持向量机的紫外吸光法COD 检测仪研制 [J]. 中国仪器仪表. 2008(02)：79-82.
[6] Singh K P, Basant N, Gupta S. Support vector machines in water quality management[J]. Analytica Chimica Acta. 2011, $703(2)$ : 152-162.

[7] 陈华凌, 陈岁生, 张仁政. 基于Zigbee无线传感器网络的 水环境监测系统 [J]。仪表技术与传感器. 2012(01): 71-73.

[8] Jiang P, Xia H, He Z, et al. Design of a Water Environment Monitoring System Based on Wireless Sensor Networks [J]. Sensors. 2009, 9(8) : 6411-6434.

[9] Zulhani Rasin, Mohd Rizal Abdullah. Water Quality Monitoring System Using Zigbee Based Wireless Sensor Network[J]. International Journal of Engineering \& Technology, 2009, 9(10), 24-28.

[10] Argent R M, Perraud J M, Rahman J M, et al. A new approach to water quality modelling and environmental decision support systems[J]. Environmental Modelling \& Software. 2009, 24(7): 809-818.

[11] 翟战强, 蔡少华. 基于GPRS/GPS/GIS的车辆导航与监控系 统 $[J]$. 测绘通报. 2004(02)：34-36.

[12] 徐兴, 岳学军, 林涛. 基于ZigBee网络的水环境无线监测 系统设计 [J]. 华南农业大学学报. 2013 (04)：593-597.

[13] 杨成成, 汪雄海. 城市污水排放实时监控界面及查询优化 设计 $[J]$. 机电工程. 2008(12)：70-73.

[14] GE YING-CHUN, LI XIN, Huang CHUN-LIN, et al. A Decision Support System for irrigation water allocation along the middle reaches of the Heihe River Basin, Northwest China [J]. Environmental Modelling \& Software, 2013, 47: 182-192.

[15] 刘卉, 汪禁华, 王跃宣, 等. 基于无线传感器网络的农田 土壤温湿度监测系统的设计与开发 $[\mathrm{J}]$. 吉林大学学报 (工 学版). 2008(03) : 604-608.

[16] GA0 CHA0, DU JIN-LING. Adaptive heartbeat mechanism for meteorology operation command system based on GPRS[C]. Science and Engineering, 2009, 2522-2525. 